\title{
Report and literature review on two cases with different kinds of Creutzfeldt-Jakob disease
}

\author{
CHUNHUA TANG and CHANGYUE GAO
}

Department of Neurology, Daping Hospital, The Third Military Medical University, Yuzhong, Chongqing 400042, P.R. China

Received April 20, 2017; Accepted September 8, 2017

DOI: 10.3892/etm.2017.5481

\begin{abstract}
Creutzfeldt-Jakob disease (CJD), also known as corticostriate spinal degeneration, subacute spongiform encephalopathy or infectious spongiform encephalopathy, is a type of degenerative disease of the central nervous system caused by prion protein ( $\mathrm{PrP}$ ) infection, which is the most common type of human PrP disease. CJD is genetic and infectious, and is one of the most common causes of rapid progressive dementia with rare clinical occurrence. Herein, we report the clinical conditions of 2 cases of patients with different type of CJD we treated and followed up recently, and a review of relevant literature. The patient in case 1 was admitted due to 'dizziness with hypomnesis, and mental and behavior disorder'. He was considered to suffer from a central nervous system infection - a viral encephalitis, but one month later, a repeated cranial MRI showed lace sign of bilateral frontotemporal parietal lobe in DWI sequence, an AEEG indicated periodic synchronous discharge, and the detection of cerebrospinal fluid 14-3-3 protein was positive. It was suggested to be diagnosed as the sporadic CJD. The patient in case 2 was admitted because of 'progressive hypomnesis'. Cerebrospinal fluid 14-3-3 protein detection was negative, but the V203Irelated mutation was found in the PRNP gene detection. The patient was suggested to be diagnosed as genetic CJD. Both patients died in a short time. An earlier diagnosis can provide a time window for treatment, and avoid unnecessary transmission in hospital, as well as doctor-patient dispute.
\end{abstract}

\section{Case report}

Clinical data

Case 1. The patient, male, 49 years old, was admitted to the Department of Neurology, Daping Hospital, The Third

Correspondence to: Dr Changyue Gao, Department of Neurology, Daping Hospital, The Third Military Medical University, 10 Changjiang Branch Road, Yuzhong, Chongqing 400042, P.R. China

E-mail: gaocy2017@163.com

Key words: Creutzfeldt-Jakob disease, spongiform encephalopathy, prion
Military Medical University on 2016-02-13 due to 'dizziness with hypomnesis for one month, and mental and behavior disorder for three days'. The patient suffered from dizziness and lethargy with myopia and hypomnesis after catching a cold one month before admission. Three days previously, his family found that the patient suffered from balderdash, which was sometimes soliloquized and smirked alone. Physical examination relieved that the patient had a clear spirit and smooth communication, but gave irrelevant answers with hypomnesis; The calculation ability was poor. There was no special abnormality in the examination of the nervous system. After admission, cranial MRI suggested that a change happened in the left parietal lobe and bilateral caudate nucleus heads; infection was not excluded. Cephalic and cervical CTA displayed no obvious abnormality. The initial pressure in the lumbar puncture was $130 \mathrm{~mm} \mathrm{H}_{2} \mathrm{O}$. The cerebrospinal fluid routine examination, biochemical examination and virus DNA examination, and three-major staining examination showed that the protein $(0.57 \mathrm{~g} / \mathrm{l})$ was in the normal range. EEG displayed moderate abnormality. The blood routine examination, hepatic and renal function, blood glucose, electrolytes, tumor markers, autoantibody spectrum, complete thyroid function, blood folic acid and VitB12 were normal; TPPA (-), anti-HIV (-). Blood, cerebrospinal fluid paraneoplastic markers and complete brain examination were in the normal range. It was considered that the patients might suffer from a central nervous system infection - viral encephalitis, so the patient received antiviral and symptomatic treatment.

After one week, the symptom of dizziness improved, but the hypomnesis and mental and behavior disorder were not improved significantly. The patient was discharged from the hospital upon the request of the families. After one month, the patient was admitted to the hospital again due to fever and disturbance of consciousness. According to his families, the patient suffered from the 'paroxysmal twitch' in both upper limbs. Physical examination revealed that the body temperature was $38.5^{\circ} \mathrm{C}$, and the pulse was 140 times $/ \mathrm{min}$. Patient displayed moderately coma, suspiciously positive neck resistance, abolition of orbital tension reflex, isometric and circular bilateral pupils, sensitive to light reflex, and bilateral pathological signs (-). A cranial MRI was conducted again and showed that the intracranial abnormal DWI signal were more than before. AEEG indicated periodic synchronous discharge. The lumbar puncture examination was performed again and the initial pressure was $160 \mathrm{~mm} \mathrm{H}_{2} \mathrm{O}$, and the 

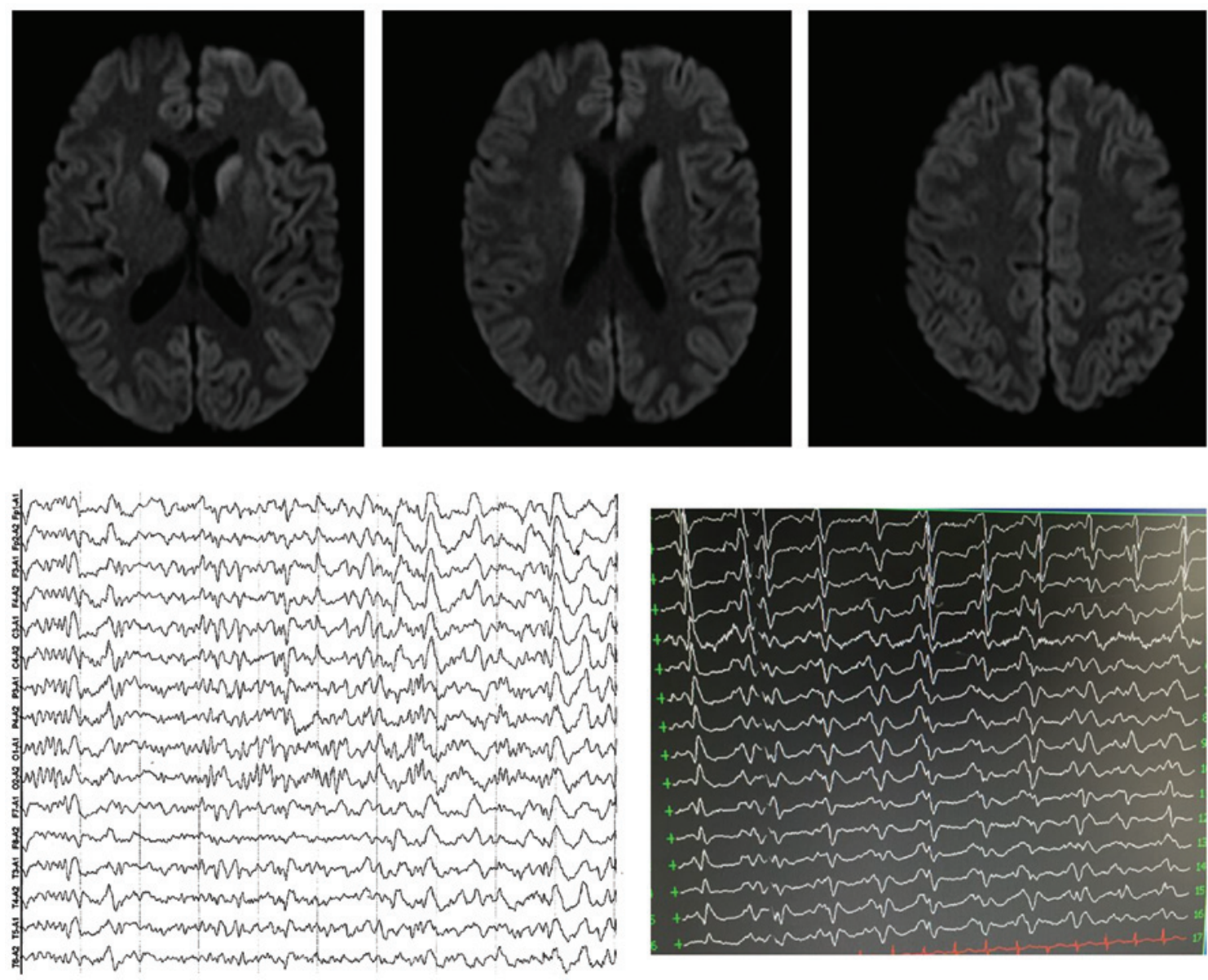

Figure 1. The bilateral caudate nucleus, globus pallidus and corona radiate region had obviously high signal in head DWI sequence in the first admission, and lace sign was seen in bilateral frontotemporal parietal lobe. The lower left image shows the moderate abnormality in routine EEG in the first admission, and the lower right image shows the paroxysmal periodic discharge in VEEG in the second admission.

cerebrospinal fluid routine examination and biochemical examination suggested that the protein $(0.62 \mathrm{~g} / \mathrm{l})$ had no significant abnormity. The cerebrospinal fluid specimens were submitted to the Chongqing Center for Disease Control (CDC) for inspection, the results suggested that the cerebrospinal fluid 14-3-3 protein was positive, and the PRNP gene detection showed that the 129 amino acid polymorphism was M/M type, and 219 amino acid polymorphism was E/E type. There was no mutation found related to the genetic Creutzfeldt-Jakob disease (CJD). According to the National Surveillance Program on CJD, it was suggested to be diagnosed as the sporadic CJD. The patient was followed up and deceased 2016-3-16 (Fig. 1).

Case 2. The patient, female, 61 years old, was admitted to hospital 2016-03-23 due to 'progressive hypomnesis for half a year, and exacerbation for more than 20 days'. During the past six months, the patient suffered from hypomnesis without an obvious reason, became forgetful and failed to recall what happened that day or what she said at the moment, remember the name of her friends, and got lost in a strange environment. She fell by accident 20 days before admission, and then was slow in reacting with attention deficit disorder, lack of words, and taciturnity and communication difficulties with her family. Physical examination revealed that she had clear consciousness that was slow in speech, and decreased memory, calculation ability and directional ability, but without significant abnormal signs in the nervous system. The MMSE score was 12 .

After admission, the cranial MRI suggested that the change happened in the signal in bilateral cerebral hemisphere cortices. The EEG demonstrated moderate abnormality. The blood routine examination, hepatic and renal function, blood glucose, electrolytes, tumor markers, autoantibody spectrum, complete thyroid function, blood folic acid and VitB12 were normal, TPPA (-), anti-HIV (-). The blood and cerebrospinal fluid specimens were submitted to the CDC and results indicated that the negative cerebrospinal fluid was 14-3-3 protein. The PRNP gene detection showed that the 129-amino acid polymorphism was $\mathrm{M} / \mathrm{M}$ type and 219-amino acid polymorphism was E/E type; the V203I-related mutation was found in the PRNP gene detection. According to the National Surveillance Program on CJD, the patient was suggested to be diagnosed as genetic CJD. The patient was followed up for 40 days, the disease persisted for 7 months, and then the patient died (Fig. 2).

\section{Discussion}

Creutzfeldt-Jakob disease (CJD) is reported as early as in 1929 successively by Creutzfeldt and Jakob, which is one of the most common types of PrP diseases, and appears mostly in population aged 50-70 years old in either gender. Its incubation period after infection is 4-30 years (1). CJD has the clinical features 

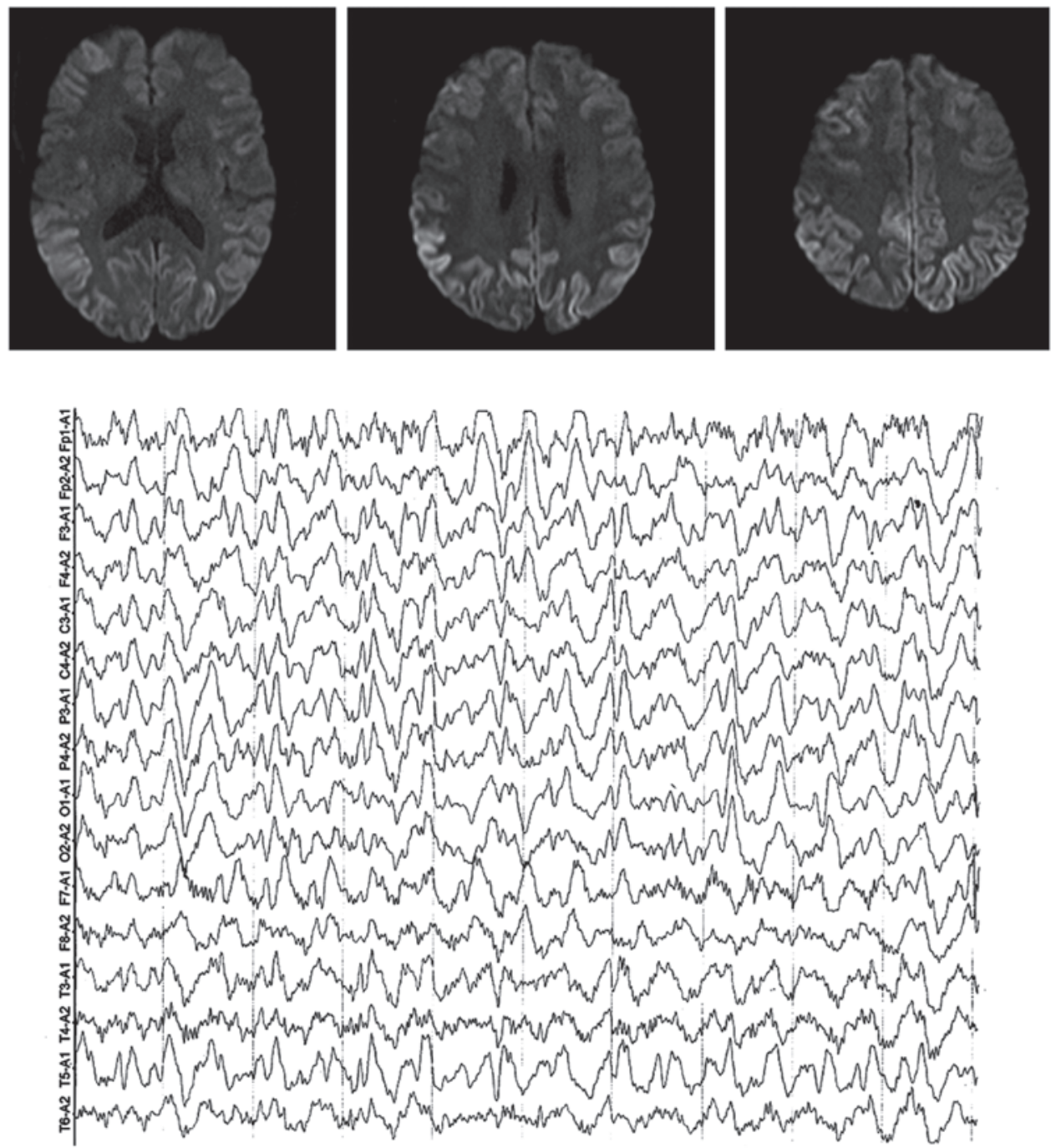

Figure 2. The cerebriform high signal in bilateral frontotemporal parietal-occipital cortex in head DWI sequence, presenting the typical 'ribbon sign'. The lower image shows moderate abnormality in routine EEG.

of rapidly progressive dementia with myoclonus, and change in EEG and MRI, which is characterized by proliferation, spongy degeneration and neuronal loss of astrocytes. Abnormal PrP, the pathogenic factor of CJD, has a very strong transmissibility, and its structure is hard to be broken by ordinary disinfection measures, thus the early recognition and diagnosis of CJD has a significant meaning for preventing its spread.

According to different causes, CJD is divided clinically into four types: sporadic CJD (sCJD), genetic or familial CJD (f/gCJD), iatrogenic CJD (iCJD) and variant CJD (vCJD). The most common type is SCJD, which accounts for $80-90 \%$ of all CJDs. The pathogenesis is often unclear, and it is generally believed that CJD is caused by PNRP gene mutations or automatic conversion of normal prion protein due to unknown causes. The disease develops quickly and the average survival period is approximately 4 months; $85 \%$ of patients die within one year (1). In this study, the first case of the patient belongs to SCJD. The early performance of SCJD is atypical, and is often difficult to identify, therefore, the patient was misdiagnosed as viral encephalitis after admission, and the antiviral treatment effect was not obvious. After one month, the disease was suddenly aggravated with myoclonus, disturbance of consciousness and typical periodic discharge in EEG, and thus, the possibility of CJD was considered. Finally, the diagnosis was confirmed through cerebrospinal fluid biomarker detection. gCJD has the family history, and the mutant PRNP is obtained through heredity, including a point mutant gene and insertional mutant gene. Now, many mutant PRNP sites have been discovered. Unlike other genetic diseases of the central nervous system, gCJD can infect others, and gCJD accounts for $5-15 \%$ in all CJDs (2).

In this study, the second case of patient belongs to this type. The patient suffered from progressive dementia, and even though there was no typical clinical manifestations, such as the myoclonus, mental disorder and ataxia, in the course of disease, and there was no specific synchronous discharge in EMG, there were typical 'lace signs' in the cerebral CT, and therefore, the possibility of CJD was considered; it was confirmed finally through genetic testing. vCJD was identified in 1996, which was caused by human consumption of animals with bovine spongiform encephalopathy. Most cases appeared in the UK, but it is also found in many other countries (3). The clinical manifestations and histology between vCJD and sCJD have obvious differences. Currently, the PrP gene in 
all patients diagnosed clearly with variant CJD, methionine homozygote located in the 129 codon was found. vCJD spread through blood transfusion was found, which suggested that vCJD can be spread through blood products. iCJD was found as early as in 1974 for corneal transplantation, and caused by the horizontal transmission between people or between people and animals; no case of vertical transmission has been found yet. The routes of iatrogenic transmission mainly include dura mater transplantation, corneal transplantation, the injection of growth hormone and gonadotropin from the pituitary in infected bodies, and the application of infected DEEG probe and neurosurgical instruments.

The auxiliary examination for CJD include pathological examination, EEG, imaging and cerebrospinal fluid examination. Pathological examination is the gold standard in the diagnosis of CJD. The typical neuronal death, proliferation of astrocytes and spongiform encephalopathy in brain tissues are observed microscopically, or the pathogenic-form $\operatorname{PrP}$ is found in immunohistochemical detection. However, the pathological examination is invasive, and combined with the strong infectivity of prion, its clinical application is limited. Therefore, other auxiliary examinations are very important to clinical diagnosis. Genetic and family history can be used as a reference in diagnosis. Moreover, the change in EEG is considered to be an important basis of clinical diagnosis; the specific periodic synchronous discharge (PSD) occurs in the middle and later periods, but there is only extensive slow-wave activity in the early or interictal period, and therefore, the EEG cannot be used as an effective early diagnostic tool. AEEG examination can improve the positive rate.

In this study, EEG had no specific performance in the first visit of the first case, however, the paroxysmal PSD was captured in video EEG in the second visit. Cranial MRI diffusion-weighted image (DWI) is both highly sensitive and specific for early diagnosis. For patients with early CJD, the abnormally high signals in the cortex and (or) basal ganglia region can appear in DWI; the abnormal banded high signal along the cortex is known as the 'ribbon signs' or 'lace signs'. In the later period of the disease, abnormally high signal in DWI will disappear. In the MRI of vCJD, the 'pulvinar nodule sign' or 'hockey stick sign' in T2WI or FLAIR sequence has a specific diagnostic significance. For the second case in this study, the typical change in DWI signal in the cortex provides strong suggestions for diagnosis. In addition, the change in multiple biomarkers of cerebrospinal fluid can be used as auxiliary evidence of CJD diagnosis, such as the protein Tau, 14-3-3 and S100, among which 14-3-3 is used more widely. The number of broken cells released into the cerebrospinal fluid increases in the onset of CJD, which has both high specificity and sensitivity. There may be a false positive in patients with subarachnoid hemorrhage, acute cerebral infarction, infectious diseases of central nervous system, frontotemporal dementia or Alzheimer's disease, and the false negative in patients with atypical subtype and variant CJD or gCJD (4). The CJD monitoring network in China is led by China CDC, and the monitoring network has covered most of provinces in China (5). Samples of both cases in this study were detected in CDC.

Geschwind et al (6) analyzed 114 sCJD patients, and found that the most common initial symptom was cognitive impairment (39\%), followed by cerebellar signs (21\%), and symptoms and signs in behavior (20\%), body (20\%), feeling (11\%), movement (9\%) and visual system (7\%). The symptoms in behavior, body and feelings, such as the headache, discomfort, and dizziness are not included in the current diagnostic criteria. The diagnosis of CJD is conducted according to the current standards of WHO (7): i) Patients with progressive dementia; ii) patients with at least two or more symptoms listed below: myoclonus, visual impairment, cerebellar ataxia, pyramid sign or extrapyramidal sign, akinetic mutism; and iii) typical EEG manifestations, namely the typical three-phase wave 1-2 times per second. Patients that meet 3 standards are diagnosed as probable CJD. Patients that meet the first 2 standards with the course of disease $<2$ years are diagnosed as possible CJD. The brain protein (cerebrospinal fluid 14-3-3 protein) detection can be used to replace the specific change in EEG. In order to make a definite diagnosis, the brain biopsy or autopsy must be conducted in order to find specific neuropathologic change. Zerr et al (8) added the imaging diagnostic basis in 2009 based on the original diagnostic criteria of the WHO. The revised new criteria increases the sensitivity of early diagnosis, which have been adopted by the US CDC. Core symptoms exist in both cases in this study, namely the rapidly progressive dementia, and there were abnormal manifestations in different degrees in head imaging examination.

The clinical manifestation of CJD and some non-PrP diseases can be rapidly progressive dementia, but the early diagnosis and treatment of some non-PrP diseases can control the progression of cognition impairment, and therefore, it is particularly important to identify this type of disease. In clinical practice, special attention must be paid to the identification between CJD and the following diseases: Alzheimer's disease, frontotemporal dementia, dementia with Lewy body, autoimmune encephalitis, paraneoplastic syndrome, central nervous system infection, and Hashimoto's encephalopathy, toxic and metabolic diseases. The standardized diagnosis process includes an analysis of medical history, physical examination and auxiliary examination item by item, which is helpful in diagnosis. The auxiliary examinations, especially the early change in head MRI, provide an important basis for identification and diagnosis. However, very few patients still cannot be diagnosed clearly even after comprehensive and systematic evaluation.

CJD has a certain infectivity, and therefore, patients must be isolated and medical personnel should be protected once it is confirmed. Daily treatment for patients are as follows: i) The special ward or negative pressure ward is not needed, but a single room is the best (convenient for treatment and care); ii) the patient's excretion and urine do not need special disinfection treatment; and iii) the ward does not need the special disinfection treatment after the patient's admission or discharge, however, the place contaminated by patient's blood or other samples can be soaked in $\mathrm{NaClO}$ containing $20,000 \mathrm{ppm}$ free chlorine or $2 \mathrm{~mol} / \mathrm{l} \mathrm{NaOH}$ for $1-2 \mathrm{~h}$ for inactivation. Protection for medical personnel are as follows: i) Try to avoid direct contact with CJD patient's blood; ii) avoid direct perforating wound in nursing, examination and treatment; iii) rinse immediately with plenty of water in the event of an accident; and iv) wear gloves in daily contact with the patient's body, but the respiratory protection is not needed. For 
treatment with close contacts, no isolation or clinical observation is needed. Principles of handling instrument and patients' supplies: i) Try to use disposable instruments and supplies, instrument contacting surface needs no special disinfection, but instrument contacting tissues must receive special disinfection; ii) the reusable non-disposable metal instrument can be inactivated by high pressure steam at $134-136^{\circ} \mathrm{C}$ for $1-2 \mathrm{~h}$, and the one-time sharp instrument can be soaked in $2 \mathrm{~mol} / \mathrm{l}$ $\mathrm{NaOH}$ for $1 \mathrm{~h}$ and incinerated in the appropriate container; iii) the patient should try to use disposable supplies, and those supplies must be incinerated after patient's death; clothes and bedding polluted by blood should be incinerated.

CJD is a fatal disease, and there is no effective cure yet. The clinical treatment mainly includes symptomatic and supportive treatment and intensive care. The main pathogenic factor is the transformation of PrPC to PrPSc, and thus, stopping the transformation of PrPSc has become the potential therapeutic target (9). Research hotspots currently include drug therapy, immunotherapy and RNA interference. In this study, two cases of patients actively received symptomatic and supportive treatment, but both of them died soon due to the lack of efficient drug.

CJD has attracted more and more clinical attention due to its atypical clinical manifestations and the lack of early diagnostic method and effective treatment. We should clarify the diagnosis with the aid of related auxiliary examinations, especially imaging and cerebrospinal fluid examination. An earlier diagnosis can provide a time window for treatment and avoid unnecessary infection and doctor-patient dispute. Currently, further research on anti-PrP drug, immunotherapy and gene therapy is the development direction of successful treatment, and further in-depth study is needed.

\section{References}

1. Sikorska B, Knight R, Ironside JW and Liberski PP: Creutzfeldt-Jakob disease. Adv Exp Med Biol 724: 76-90, 2012.

2. Rosenbloom MH and Atri A: The evaluation of rapidly progressive dementia. Neurologist 17: 67-74, 2011.

3. Ironside JW: Variant Creutzfeldt-Jakob disease: An update. Folia Neuropathol 50-56, 2012

4. Zanusso G, Fiorini M, Ferrari S, Gajofatto A, Cagnin A, Galassi A, Richelli S and Monaco S: Cerebrospinal fluid markers in sporadic Creutzfeldt-Jakob disease. Int J Mol Sci 12: 6281-6292, 2011

5. Gao C, Shi Q, Tian C, Chen C, Han J, Zhou W, Zhang BY, Jiang HY, Zhang J and Dong XP: The epidemiological, clinical, and laboratory features of sporadic Creutzfeldt-Jakob disease patients in China: Surveillance data from 2006 to 2010. PLoS One 6: e24231, 2011.

6. Geschwind MD, Shu H, Haman A, Sejvar JJ and Miller BL: Rapidly progressive dementia. Ann Neuro 164: 97-108, 2008.

7. WHO: Global surveillance, diagnosis and therapy of human Transmissible Spongiform Encephalopathies: Report of a WHO consultation. Geneva, Switzerland, 9-11 February 1998. World Health Organization, Geneva, Switzerland, 1998.

8. Zerr I, Kallenberg K, Summers DM, Romero C, Taratuto A, Heinemann U, Breithaupt M, Varges D, Meissner B, Ladogana A, et al: Updated clinical diagnostic criteria for sporadic Creutzfeldt-Jakob disease. Brain 132: 2659-2668, 2009.

9. Marandi Y, Farahi N, Sadeghi A and Sadeghi-Hashjin G: Prion diseases - current theories and potential therapies: A brief review. Folia Neuropathol 50: 46-49, 2012. International (CC BY-NC-ND 4.0) License. 